\title{
Crowd Interest Mapping to Assess Engagement
}

\author{
https://doi.org/10.3991/ijoe.v18i02.25445 \\ Gustavo Assunção( $\left.{ }^{\square}\right)$, Bruno Patrão, Paulo Menezes \\ Department of Electrical and Computer Engineering, University of Coimbra, Coimbra, Portugal \\ gustavo.assuncaodisr.uc.pt
}

\begin{abstract}
Emotionally pleasant experiences trigger repetition in humans whilst their emotional opposite lead to avoidance/refusal of activities. Human interest, a key factor in everyday human life, can be quite useful in the evaluation of participant engagement on activities and their optimization, so as to maximize interest and motivation. A glaring issue however comes from subtle demonstrators associated with human interest, which converge onto its complex assessment/quantization. Yet, there is an inherent correlation between human interest and its provoked emotional response which can be explored in tandem with emotion recognition for the development of an engagement metrics tool. Such a mechanism would be highly beneficial for the improvement of several activities involved with learning and security, by enabling precise control over participant enthusiasm. In this paper we present an interest mapping technique which provides the user with spatio-temporal information extracted from a participant crowd. The technique aims to extract emotional cues from participant facial data, assessing its spatial and temporal distributions over the course of scenarios such as therapy sessions and lectures. The goal is to demonstrate where and when activities must be improved in order to retain attention, maximize efficacy and assure emotional pleasantness in participants. For validation, this study makes use of data collected over a college lecture so as to provide readers with a real demonstration of the technique's advantages.
\end{abstract}

Keywords-emotion recognition, machine learning, human perception

\section{Introduction}

Emotion is extremely influential to our behavior in any situation, holding a pivotal role in human life. For instance, [1] demonstrated how emotion acutely modifies our physiological responses to different situations. This phenomenon stems from the nature of emotion as a trigger, developed for responding appropriately to situations that may occur in our environments [2], whether social or not. Though its study has been relatively intensive over the last century, with several models being presented such as the emotional wheel [3] or the Pleasure-Arousal-Dominance (PAD) [4], its aspects as a regulatory metric for therapeutic or rehabilitation procedures has seldom been given attention by the research community. This demonstrates a blatant disregard for the link between triggering/retardant emotionality and attention/interest. This notion is supported by several views in the field of psychology and behavioral studies, such as 
[5] and [6]. Thus, it motivates the development of applications which may take advantage of this information. For instance, a tool could be developed for therapy, analyzing sessions once finished so as to identify which sections worked best for each patient and remove ineffective ones. Similarly, a platform could be developed for teachers to identify possible sources of student disinterest in their class plan.

In terms of rehabilitation, one may argue maintaining a patient's interest and motivation in scenarios where these are lacking is of paramount importance for successful treatment. This is especially true for cognitive rehabilitation related to depressive issues or behavioral disorders (e.g. alcoholism, mania, assorted phobias), commonly dealt with in group therapy sessions [7], [8]. Enabling a caregiver to visualize how patient interest and motivation are progressing over time allows for posterior session enhancement and greatly reduces the chances of patients abandoning therapy. Other scenarios where participant interest can be a valuable metric include lectures, during which sparking and preserving audience engagement is key for effective conveyance of information. Moreover, monitoring large audiences through wearable devices, as in [9], might not always be a feasible option. Yet, enabling lecturers to correlate informational flow with crowd interest and evolvement can help optimize conveyance and reduce accumulated pressure/frustration. Even more so during current pandemic times, with lectures switching to a virtual setting. An engagement feedback mechanism becomes a necessity, in lieu of the physical response to which speakers are accustomed. Furthermore, reduction of public speaking anxiety is another incentive for this work. As pointed by [10], artificial audiences in virtual reality (VR) have been used to help struggling lecturers. People are able to develop a sense of confidence and control over their instability, resulting in more successful real-world lectures. Nevertheless, virtual crowds generally enact premade situations rather than mimicking actual lectures. Oppositely, a real correlation can be realized between lecturer and students through interest mapping, useful for accurate response emulation in VR.

In this work we present an interest mapping technique based on the recognition of archetypal emotions based on facial expression. Our goal is to improve group therapy as well as lecture scenarios, providing speakers with an engagement metric. By periodically extracting the emotional state of each participant, based on facial analysis, a score is generated from the correlation between those states and human interest. This is done in order to formulate a visual representation of human interest over a crowd of participants, depicting its flow by morphing shape and texture over time. Though not many techniques have attempted crowd interest mapping in literature, a technique based on face and body motion was presented in [11], for predicting movie ratings in theatres. Instead, we aim for the proposed tool to be useful in teaching frameworks.

This paper is divided accordingly: section 2 presents an overview of recent work and is followed by section 3 where we detail the methodology used, specifying how data was gathered and posteriorly employed in our mapping technique using machine learning. Section 4 then reviews the carried out experiments and displays the obtained results, while section 5 provides insight into those results as well as a corresponding critique. Finally, section 6 provides a conclusion on the topic in addition to a summary of future work. 


\section{Related work}

This section is branched into a set of subtopics. First, detail is provided on emotion theory and modelling in order to frame the key concepts of the developed technique. Following that, we overview recent research aimed at human interest measuring and mapping using computational techniques (e.g. machine learning). Subsequently, an overview is provided on studies addressing the importance of maintaining patient/participant motivation and interest for successful therapy/learning. As such, we overview techniques attempting to achieve this goal.

\subsection{Emotion theory and modeling}

While emotion is a topic of continuous disagreement among researchers, certain aspects are widely accepted. The most basic of these is emotion being treated as either categorical or dimensional. The former considers a set of archetypal emotions capable of representing most or all human states. These are reduced to include only 6 to 8 emotions, typically happiness, sadness, fear, anger, surprise and disgust, with neutrality corresponding to lack of emotion. The emotional wheel [3] or the circumplex model of affect [29], two of the most common categorical emotion models, exemplify this by delimiting states as angular measures. On the other hand, dimensional perspectives generally assume a minimum of two scores (valence and arousal) to represent human states. Here, each emotion makes up a point in $\mathrm{N}$-dimensional space where $\mathrm{N}$ refers to the number of scores, meaning there is an infinite number of possible states. One of the more recent dimensional models is the PAD model [4], which considers a third axis pertaining to the dominance factor of each emotional state. Moreover, it is not uncommon to devise a matching between categorical and dimensional models so as to take advantage of the two. This can be achieved as archetypical emotional states are represented by both types of models. Incidentally that is performed in this work, where only a set of archetypical states is considered to simplify the full range of crowd emotions, though placed over a colored dimensional space to better visualize its progression.

Modeling facial expressions as emotion is also not a trivial task. However, the correlation between certain emotional states and activations of certain facial regions is self-evident during human interaction. This prompted Ekman and Friesen to develop the Facial Action Coding System (FACS) [12]. By comparing facial expressions in humans from different cultures, these researchers isolated specific points of the human face (the action units) and associated combinations of these with the emotional states demonstrated by the subjects. This system has since become a standard for facial expression analysis and as such is employed in our work to extract emotion from a crowd of participants.

\subsection{Human interest metrics}

Human interest and attention have long been subjects of study in the field of Psychology and behavioral analysis. Yet only recently, with technological development 
and the advent of big data approaches, has this topic been taken into consideration by engineers and computer scientists throughout. To exemplify, in [13] a headset was used to capture EEG-based emotional data parallelly with participant self-surveyed level of interest while being shown educational multimedia content for correlation with learning success. Relatedly, EEG was also employed in [14] where data acquired from students during a college lecture was used to classify the degree of situational interest of each participant, using conventional classifiers such as kNN. Other methods for interest detection have involved technologies such as eye tracking [15] and manufactured expression/pose metrics [16], though less frequently.

Applications and research on automated correlation between emotion recognition and human-interest levels are rather scarce. That being said, works more strongly linked with our own include [17], where a framework is presented for creating worldwide and temporally distributed interest maps of specific words/topics based on emotional analysis of tweets, and [18], where the authors detect interest from facial analysis through a constructed recombination of the typical correspondences between FACS and the archetypal emotions psychologically thought to cause that interest. These approaches however are lacking in robustness and cannot realize our proposed objectives to an adequate extent.

\subsection{Interest and motivation as success factors}

The correlation between successful task accomplishment and level of interest/motivation is a topic commonly addressed by Psychology. In fact, several studies have attempted to verify this relationship, with [19] noting increased motivation and adherence to cognitive rehabilitation sessions by psychotic patients who underwent an initial motivational interviewing preparation, in addition to predicting number of attended sessions based on assessment of motivational level. In education scenarios the observations are similar, with student interest playing a major role in learning and current schooling approaches shifting more towards fomenting motivation and sparking curiosity [20] rather than incentivizing conventional repetition.

Furthermore, the influence of emotion over task interest and motivation is a widely accepted fact, supported for instance by its function as a trigger of curiosity and reassessment of known data [21], as well as by the role of epistemic emotion (e.g. surprise) on regulation of knowledge exploration [22]. Were these reasons not enough, emotional vitality has been observed to be of paramount importance for successful rehabilitation, validating its use as a treatment optimization factor [23]. Thus, it is only natural to use emotion as an index basis for measuring a participant's level of interest and engagement during some session.

\section{Methodology}

This section is meant to describe the developed method, employing crowd facial emotion recognition (FER) to obtain a visual representation of interest. A short overview of the employed FER system is first provided, followed by the overall technique. 


\subsection{Facial emotion recognition}

In order to infer conclusions based on crowd emotion, and considering how participants are most often silent and still, deeming speech of body emotion recognition inadequate, FER was employed as the best alternative. To this end, FACS was used in order to objectively represent facial expressions for posterior correspondence with underlying emotion. The technique of [24] uses a VGG16-based CNN to extract FACS action units and assign an emotional state, yielding almost a good accuracy rate with only 12 action units. Evidently, in-the-wild data common of large crowds is typically cluttered, of low resolution and high noise, hindering the performance of the model. Thus, we employ deep learning for this issue as it still proves the be the most adequate approach. Moreover, as FER is only a module of our overall technique, it is always possible to update the deep model whenever others are made available, if proven to be more robust and considerate of the variance in FACS-based emotional representations.

\subsection{Interest mapping}

The main idea behind this technique is the well-established concept of emotional contagion [25], which supports the notion of human emotional state directly or indirectly affecting that of the surrounding group or vice-versa, due to the reciprocating and empathic nature of human behavior. A visualization of this process is shown in Figure 1. This has been empirically demonstrated by a panoply of studies, such as [26] or the more recent [27], and motivated our developed visualization technique.

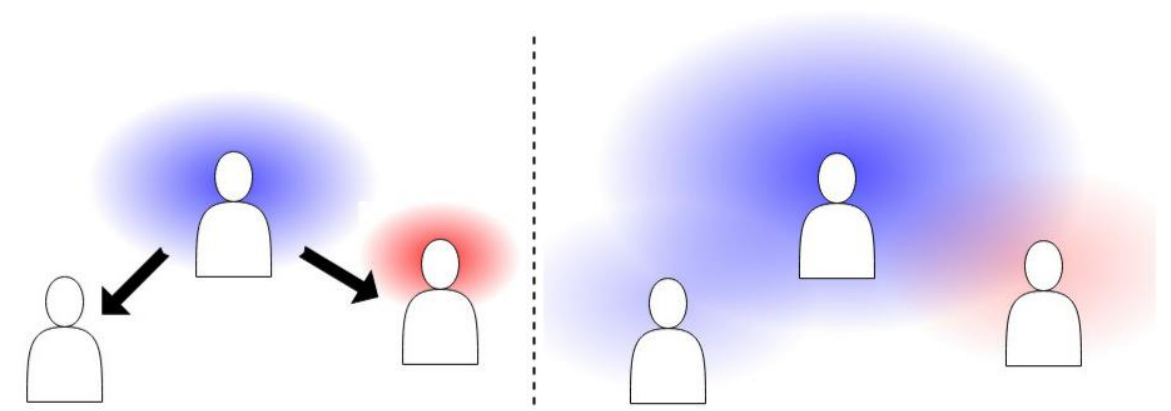

Fig. 1. Emotional Contagion, where a sad individual (blue aura) approaches two others: neutral (no aura) and slightly angry (red aura). Through interaction, the left and right ones are influenced, integrating what emotional information they perceive (subconsciously or not) - left with traces of sadness and right shaded to a combo of sadness and anger

Evidently, and sustained by the related work revised above, influence over a person's state will also affect their interest and motivation levels, leading to gradual changes in these similarly to emotionality itself. Consequently, the mapping technique focused on recreating this emotional contagion effect in a visual manner as well as on projecting the intervening elements based on an emotion-interest correlation metric. To begin with, and taking into advantage how a person's face is individually pin- 
pointed in a crowd, the position, face detection confidence and average confidence of incurring FACS actions units of each recognition are used to generate a colorized aura around the corresponding person. This elliptical representation is aligned with the face's position and elongated accordingly with the face detection confidence, as a more clearly visible face naturally leads to a more acute emotional contagious effect on its surrounding peers. As for the hue of the generated aura, it is regulated by the activated FACS units through a cumulative average of their probabilities. This value factors in the alpha channel calculation for each aura image, allowing for the representation of more intense or milder colors at the epicenter of the aura. These details are shown in Figure 2, while a flow diagram of the overall technique is shown in Figure 3.

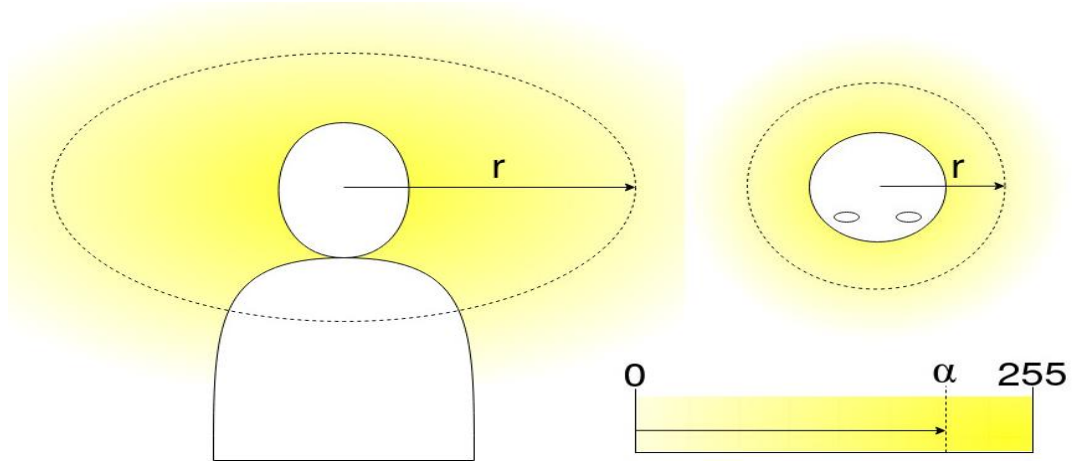

Fig. 2. Visual formulation of an emotional aura surrounding the person with the corresponding state. Here, $r$ represents the factor by which the aura is elongated and $\alpha$ the max value of the alpha channel calculated for that same aura

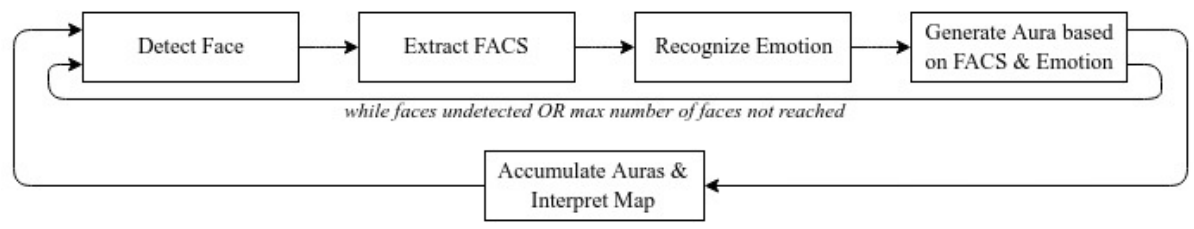

Fig. 3. Flow diagram of the continuous interest mapping and FACS + emotion-based interpretation process, as previously described

Following the formulation of auras for each face and underlying emotion, all these objects are overlapped together. In accordance with emotional contagion, this combination of colors and corresponding intensities creates an accurate visual representation of the overall emotional disposition over the participant crowd. Moreover, the intensity of this effect is congruent with participant proximity. Finally, a mapping is formed over the crowd, which freely modifies its shape, intensity distribution and color over time, according to how a person's emotional state is constantly evolving. In order to achieve smoother state transitions and reduce a flickering on the mapping, auras are formulated every fps/ 6 frames, where fps corresponds to the number of frames per second for the recording, through a cumulative sum and normalization of 
each person's color values. In accordance with the employed FER model, 6 emotional states were considered for the mapping technique, each receiving a distinct RGB color as noted in Table 1.

Table 1. Color scheme assigned to the considered emotion set, as well as symbolic valencearousal scoring based on the well-established circumplex model of affect [29]

\begin{tabular}{|l|c|c|c|}
\hline \multicolumn{1}{|c|}{ Emotion } & Color & Valence & Arousal \\
\hline Happiness & Yellow & Strong Positive & Strong Positive \\
\hline Sadness & Blue & Strong Negative & Weak Negative \\
\hline Fear & Green & Strong Negative & Weak Positive \\
\hline Anger & Red & Strong Negative & Strong Positive \\
\hline Surprise & Turquoise & Weak Positive & Strong Positive \\
\hline Neutral & White & - & - \\
\hline
\end{tabular}

The bridge connecting emotionality to an interest metric was based on the different types of motivation caused by distinct valence-arousal configurations such as those of individual emotional states. Thus, emotions may be qualitatively mapped onto a valence-arousal scale, based on their characteristics, and in turn correspond with perceived levels of either autonomous or controlled sub-types of motivation as in [6]. Specifically, the authors reported greater intrinsic motivation in people experiencing positive high-valence emotions, whilst neutral low-arousal states were seemingly deactivating and causing demotivation. Another study by the same authors [28] also noted low motivation in negative valence scenarios, which increased as people became more relaxed and experienced feelings of happiness. In [5], the authors observed interest decreased simultaneously to an arousal drop - valence raise combo after tasks were completed. Their findings also matched common sense, showing that lower interest levels are correlated with valence decreases, for instance when tasks become unattractive to participants. Additionally, arousal increases were matched with raise of interest when performing new or urgent tasks.

Naturally, considering the colorized emotional mapping method already described, the next step is to interpret it in terms of interest. Therefore, an appropriate legend/color bar must be created. Based on the observations of past studies, and by subdividing the known circumplex model of affect [29], the color wheel legend shown in Figure 4 was created for interpreting the emotional maps as indicators of interest/motivation levels. The lower right corner of the color wheel was left blank as no emotional state pertaining to that specific zone of valence-arousal score combination was considered in our approach. 


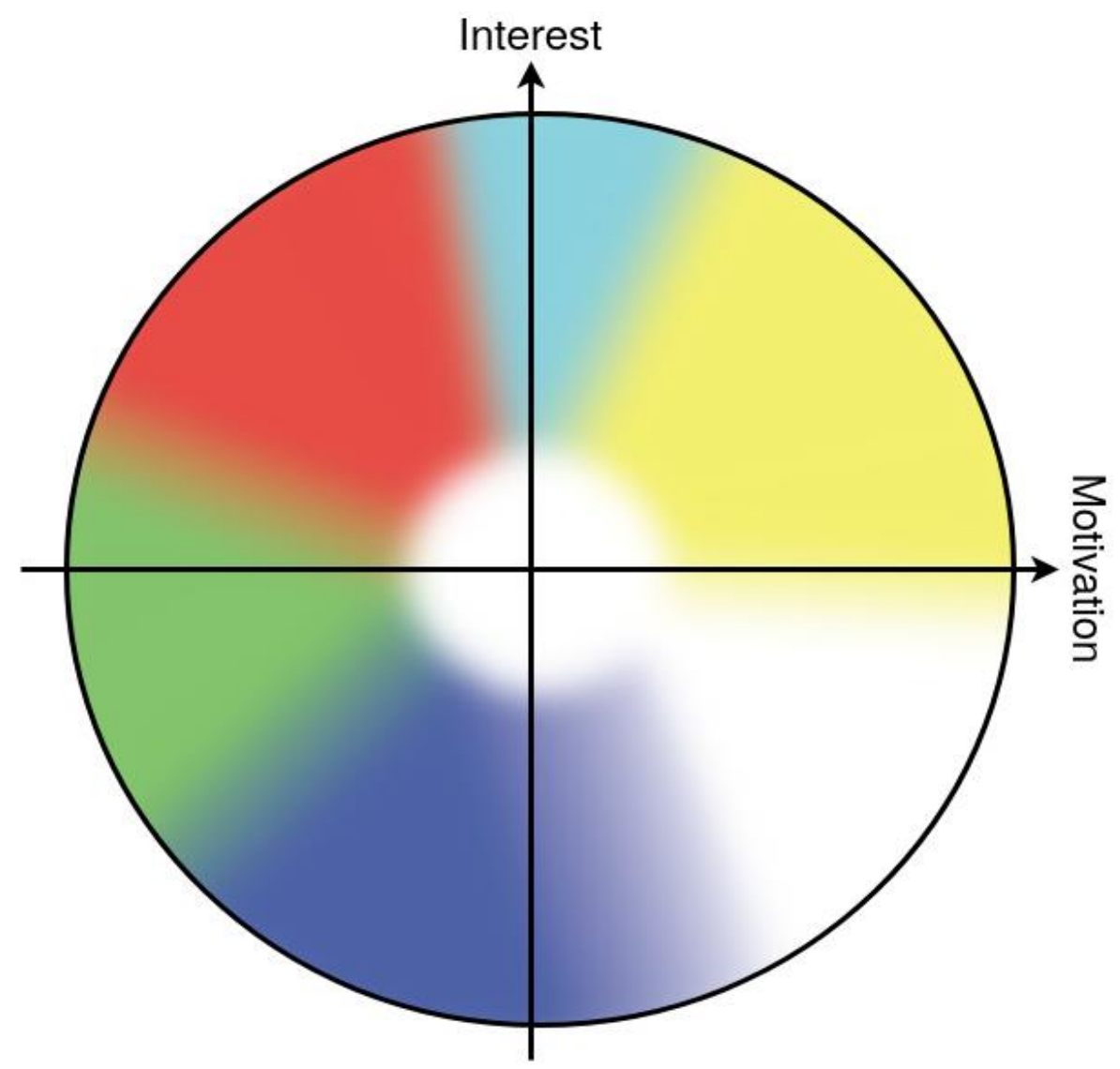

Fig. 4. Visual Devised color wheel for interpretation of the emotional maps in terms of interest-motivation levels, based on the circumplex model of affect described in [29]

\section{Experiments}

In order to evaluate the efficacy of the proposed technique an exemplary set of data was required. This could be obtained either during a group therapy session, college lecture or presentation where a considerable number of faces were visible in the crowd and spaced well enough to produce acceptable visual results. Unfortunately, given the pandemic scenario the world is currently facing, it was infeasible to obtain data as initially planned. As an ingenious alternative, a recording of a virtual college lecture was made and used for experimental purposes. The lecture lasted approximately 1 hour and was on specific topics of Computer Graphics never before taught to the students, so as to more accurately assess their interest. There was a total of 12 student attendants in addition to the lecturer, all of whom signed an informed consent agreement regarding being recorded and participating in this experience. 
The technique was applied directly on the lecture recording without any preprocessing whatsoever. The created interest-motivation color wheel was used as an interpretation tool of the visual representations. On Figure 5, a set of distinct lecture scenarios is shown, which are already expected to generate different responses in the audience. For each presented scenario, the mappings were overlapped with the corresponding lecture video segment and samples of this overlap were screenshotted from random, evenly spaced timestamps. The goal was to observe whether or not the interpretation of the created mappings matched these expectations.
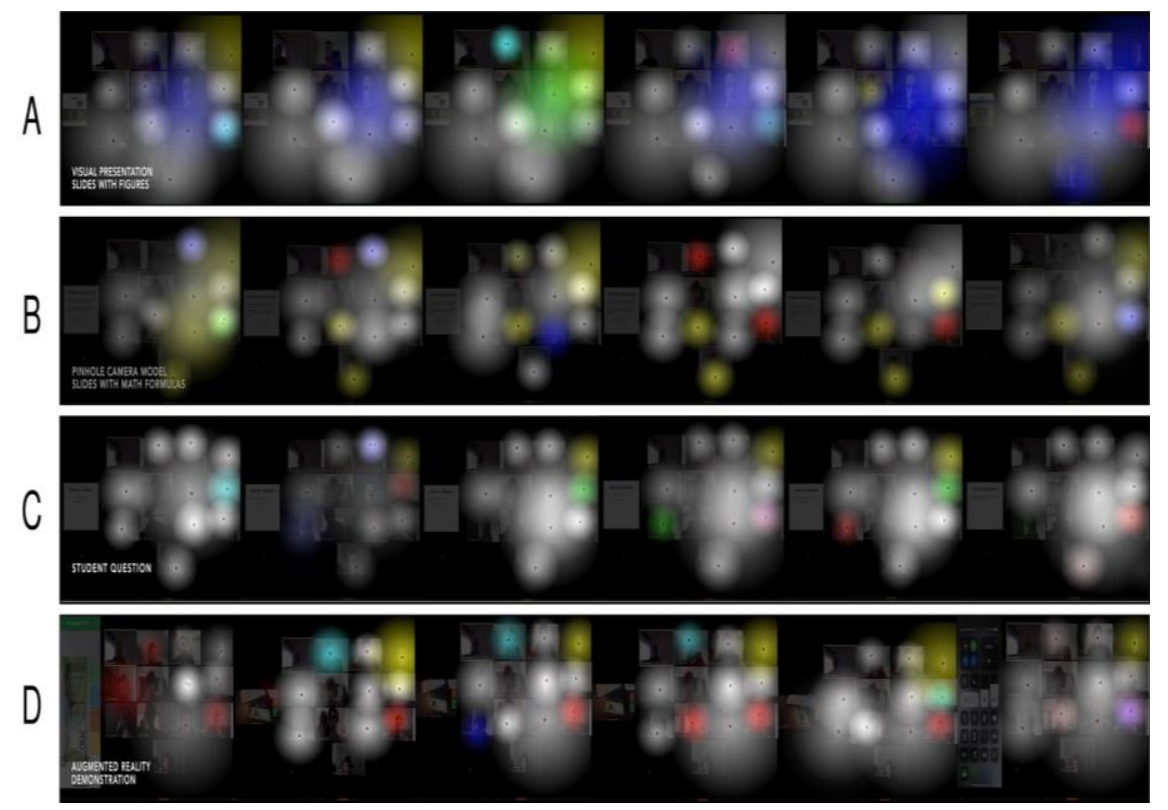

Fig. 5. Four distinct lecture scenarios represented by randomly extracted overlapped frames of the interest-motivation mappings and their corresponding lecture video settings. These scenarios are the following: A - Welcoming and Class Introduction; B - Pinhole Camera Theory Explanation; C - Student Question and Interaction; D - Visual demonstration of AR

A mere initial observation of the overlapping results in Figure 5 is sufficient to notice significant differences between the four scenarios A, B, C and D, both in terms of color and aura sizes. In some cases, a particular color is overly abundant, while in others certain parts of the image show greater aura sizes than in other areas, despite no specific color having majority. These observations are discussed in the following section. 


\section{Discussion}

As can be seen in the previous section, the different lecture scenarios did indeed produce distinct interest-motivation mappings generated using emotion recognition and a valence-arousal based interpretation. Naturally the technique also has a downside. For example, the face detector may fail to detect all faces in a crowd due to poor resolution or occlusion, or contrastingly detect false positives, which may affect the posterior processing stage. Nevertheless, these situations do not occur frequently thanks to the deep-learning nature of the model and periodical averaging out of frame results. In addition to this, the small number of participants in this experiment also affects mapping resolution. A greater number of participants would certainly yield a perceptibly better map. However, given the current pandemic circumstances, testing with greater crowds is postponed to safer times.

Addressing now each lecture scenario specifically, in situation A blue is the predominant color. This indicates low interest and somewhat low to null motivation. As expected, generally the common and unbiased student is initially uninterested in topics they have never heard of before up until the moment when some particular aspect of those topics sparks personal interest. Further, stagnant motivation levels are congruent with students who deal with daily classes or lectures. A more compelling case is presented in situation $\mathrm{B}$, as interest-motivation levels are exceedingly particular to each participant rather than a more robust consensus existing among the crowd. Due to the theoretical nature of this situation, it is expected for opinions to vary as people develop their own opinions and become more or less interested, hence generating auras of different colors. Yet, red and yellow are the predominant colors, indicating high interest but opposing levels of motivation in different participants, also congruent with common sense.

Situation $\mathrm{C}$ is a perfect example of the system's functioning. As a particular student (central-right dot) asks a question, most of his colleagues look away uninterested, as opposed to himself who moves closer to his screen/camera due to increased interest. The system captures this well as some colleague auras reduce in size or disappear completely as their faces become harder to recognize, whilst the questioner's aura significantly increases. This led us to conclude, in virtual scenarios such as the one tested, that aura size could also be a good indicator of participant interest. Lastly, in situation D, results are somewhat similar to those of situation B, as the augmented reality demonstration produces different levels of interest in each participant. However, the predominant colors are again red and yellow with an occasional turquoise, indicating high levels of interest in the presentation, but varying degrees of motivation which naturally depend on each person and how their state may be after over one hour of lecture.

In terms of comparison with similar works, ours differs in terms of robustness and visualization. In [17], for instance, text may not always convey the correct emotion or allow for an accurate level of interest to be extracted. The work presented in [18] does not have this issue. However, this latter work does not perform a temporal or spatial analysis of participant interest nor does it provide users with visual metrics. 
As a final remark, it is important to note how the same people tend to maintain the same interest-motivation levels overall throughout a situation, but suffer change when transitioning to different situations. This is key in asserting how the devised technique was successful in capturing interest-motivation levels to some degree, despite the relatively small size of the crowd.

\section{Conclusion}

This study focused on the creation of a crowd interest mapping tool, through development of a system based on facial emotion recognition. The technique took advantage of the emotional contagion effect to generate a visual representation of how emotional states and corresponding intensities flow in a crowd, using colorized personal auras. These were interpreted as interest/motivation metrics using psychological findings on the correlation between different valence-arousal scores and the interest/intrinsic motivation effects they trigger in people. Experimentally, the method favored well in a virtual college lecture. Here it successfully differentiated situations in a visual manner, where participant interest-motivation was retroactively expected to vary. The experiment validated the approach, as it allowed for posterior improvement of the presented content and method of conveyance.

In the future, the technique will be tested in physical scenarios as initially planned, after which pose estimation and other related factors will be incorporated for a more accurate interest metric and display. Furthermore, we intend to deploy the method as a standalone and easy to use tool for therapeutic or lecture-like improvements.

\section{$7 \quad$ Acknowledgment}

This work has been partially supported by Fundação para a Ciência e a Tecnologia (FCT) under grant 2020.05620.BD, and OE - National funds of FCT/MCTES under project UIDB/00048/2020.

\section{$8 \quad$ References}

[1] Patrão, B, Pedro, S. Menezes, P, (2016), Human Emotions and Physiological Signals: A Classroom Experiment, Int. J. Onl. Eng. (iJOE), vol. 12, no. 4, pp. 37. https://doi.org/10.39 91/ijoe.v12i04.5098

[2] Cowie, R, Douglas-Cowie, E, Tsapatsoulis, N, Votsis, G, Kollias, S, Fellenz, W, and Taylor, JG, (2001), Emotion recognition in human-computer interaction, IEEE Signal Processing Magazine, 18(11):3280. https://doi.org/10.1109/79.911197

[3] Plutchik, R, (1980), Emotion: A psychoevolutionary synthesis. New York: Harper and Row.

[4] Mehrabian, A, (1980), Basic dimensions for a general psychological theory.Oelgeschlager, Gunn and Hain, pp 39-53.

[5] Mäntylä, M, Adams, B, Destefanis, G, Graziotin, D and Ortu, M, (2016), Mining valence, arousal, and dominance: possibilities for detecting burnout and productivity?, Proceedings 
of the 13th International Conference on Mining Software Repositories (MSR '16). Association for Computing Machinery, New York, NY, USA, pp. 247-258. https://doi.org/10.11 $\underline{45 / 2901739.2901752}$

[6] Vandercammen, L, Hofmans, J, and Theuns, P, (2014), Relating specific emotions to intrinsic motivation: on the moderating role of positive and negative emotion differentiation, PloS One, 9(12). https://doi.org/10.1371/journal.pone.0115396

[7] Ryan, Richard M., Martin F. Lynch, Maarten Vansteenkiste, and Edward L. Deci, (2011), Motivation and autonomy in counseling, psychotherapy, and behavior change: A look at theory and practice $1 \psi 7$. The Counseling Psychologist 39, no. 2, 193-260. https://doi.org/ $\underline{10.1177 / 0011000009359313}$

[8] Ogrodniczuk, John S.; Joyce, Anthony S.; Piper, William E. (2005). Strategies for Reducing Patient-Initiated Premature Termination of Psychotherapy. Harvard Review of Psychiatry, 13(2), 57-70. https://doi.org/10.1080/10673220590956429

[9] George, A, Kurup, A, Balachandran, P, Nair, M, Gopinath, S, Kumar, A and Parasuram, H, (2021), Predicting Autonomic Dysfunction in Anxiety Disorder from ECG and Respiratory Signals Using Machine Learning Models, Int. J. Onl. Eng. (iJOE), vol. 17, no. 7, pp. 143. https://doi.org/10.3991/ijoe.v17i07.22581

[10] Wörtwein, T, Morency, L and Scherer, S, (2015), Automatic assessment and analysis of public speaking anxiety: A virtual audience case study, International Conference on Affective Computing and Intelligent Interaction (ACII), Xi'an, pp. 187-193. https://doi.org/10.11 09/acii.2015.7344570

[11] Navarathna, R, Lucey, P, Carr, P, Carter, E, Sridharan, S, and Matthews, I, (2014), Predicting movie ratings from audience behaviors, IEEE Winter Conference on Applications of Computer Vision, WACV 2014, pp. 1058-1065. https://doi.org/10.1109/wacv.2014.68359 $\underline{87}$

[12] Paul Ekman and Wallace V Friesen. Constants across cultures in the face and emotion. Journal of personality and social psychology, 17(2):124, 1971. https://doi.org/10.1037/h00 $\underline{30377}$

[13] Moldovan, A, Ghergulescu, I, and Muntean, CH, (2017). Analysis of Learner Interest, QoE and EEG-Based Affective States in Multimedia Mobile Learning. IEEE 17th International Conference on Advanced Learning Technologies (ICALT), 398-402. https://doi.org/ 10.1109/icalt.2017.93

[14] Babiker, A, Faye, I, and Malik, A, (2018), EMD-Based Feature to Detect Situational Interest in Classroom Settings Using EEG, International Conference on Intelligent and Advanced System (ICIAS), Kuala Lumpur, pp. 1-5. https://doi.org/10.1109/icias.2018.854060 $\underline{8}$

[15] López-Gil, JM, Virgili-Gomá, J, Gil, R, Guilera, T, Batalla, I, Soler-González, J and García, R, (2016), Method for Improving EEG Based Emotion Recognition by Combining It with Synchronized Biometric and Eye Tracking Technologies in a Non-invasive and Low Cost Way, Frontiers in Comp. Neurosc, 10:85. https://doi.org/10.3389/fncom.2016.00119

[16] Bhattacharya, S, Dasgupta, A, Sengupta, A, and Routray, A, (2019), Interest Indices for Human Computer Interaction Applications based on Facial Image Sequences, IEEE 16th India Council International Conference (INDICON), Rajkot, India, pp. 1-4. https://doi.org/ 10.1109/indicon47234.2019.9029016

[17] Saravia, E, Argueta, C, and Chen, YS, (2015), EmoViz: Mining the World's Interest through Emotion Analysis, IEEE/ACM International Conference on Advances in Social Networks Analysis and Mining 2015 (ASONAM '15), Association for Computing Machinery, New York, NY, USA, 753-756. https://doi.org/10.1145/2808797.2808916 
[18] Kenza, B, Mohamed, B, and Hacene, B, (2015), Facial expressions of interest's emotion, 2015 International Conference on Applied Research in Computer Science and Engineering (ICAR), Beirut, pp. 1-3. https://doi.org/10.1109/arcse.2015.7338133

[19] Fiszdon, JM, Kurtz, MM, Choi, J, Bell, MD, and Martino, S, (2016), Motivational Interviewing to Increase Cognitive Rehabilitation Adherence in Schizophrenia, Schizophrenia Bulletin, 42(2) 327-334. https://doi.org/10.1093/schbul/sbv143

[20] Harackiewicz, JM, Smith, JL, and Priniski, SJ, (2016), Interest Matters: The Importance of Promoting Interest in Education, Policy insights from the behavioral and brain sciences, 3(2), 220-227. https://doi.org/10.1177/2372732216655542

[21] Tyng, CM, Amin, HU, Saad, MNM, and Malik, AS, (2017), The Influences of Emotion on Learning and Memory, Frontiers in Psychology, 8:1454.

[22] Vogl, E, Pekrun, R, Murayama, K, and Loderer, K, (2019), Surprised-curious-confused: Epistemic emotions and knowledge exploration, Emotion, 20(4), 625-641. https://doi.org/ $10.1037 / \mathrm{emo} 0000578$

[23] Barbic, SP, Bartlett, SJ, and Mayo, NE, (2013), Emotional vitality: concept of importance for rehabilitation, Archives of Physical Medicine and Rehabilitation, 94(8):1547-1554. https://doi.org/10.1016/j.apmr.2012.11.045

[24] Gerardo, PC, and Menezes, P, (2019), Classification of FACS-Action Units with CNN Trained from Emotion Labelled Data Sets, 2019 IEEE International Conference on Systems, Man and Cybernetics (SMC), Bari, Italy, pp. 3766-3770. https://doi.org/10.1109/ smc.2019.8914238

[25] Hatfield, E, Cacioppo, JT, and Rapson, RL, (1994), Emotional contagion. Studies in Emotion and Social Interaction, Cambridge: Cambridge University Press.

[26] Tsai, J, Bowring, E, Marsella, S, Wood, W, and Tambe, M, (2012), A Study of Emotional Contagion with Virtual Characters, Intelligent Virtual Agents (IVA), Springer Berlin Heidelberg, Berlin, pp 81--88. https://doi.org/10.1007/978-3-642-33197-8_8

[27] Olszanowski, M, Wróbel, M, and Hess, U, (2020), Mimicking and sharing emotions: a reexamination of the link between facial mimicry and emotional contagion, Cognition and Emotion, 34:2, pp. 367-376. https://doi.org/10.1080/02699931.2019.1611543

[28] Vandercammen, L, Hofmans, J, Theuns, P and Kuppens, P, (2014), On the Role of Specific Emotions in Autonomous and Controlled Motivated Behaviour, European Journal of Personality, Wiley, 28(5), pp. 437-448. https://doi.org/10.1002/per.1968

[29] Russell, J, (1980), A Circumplex Model of Affect, Journal of Personality and Social Psychology, 39.

\section{Authors}

Gustavo Assunção is a $\mathrm{PhD}$ candidate at the University of Coimbra, and a researcher of the Institute of Systems and Robotics in Coimbra, Portugal. His focus is on bio-like deep learning solutions, combining several topics of computational neuroscience, neurocognitive computing and the mathematical foundations of deep learning for better optimization.

Bruno Patrão is a PhD candidate at the University of Coimbra, and a researcher of the Institute of Systems and Robotics in Coimbra, Portugal. His interests include emotion regulation through immersive therapies, virtual and augmented reality environments, interactive systems and computer graphics (Email: bpatrao@isr.uc.pt). 
Paper — Crowd Interest Mapping to Assess Engagement

Paulo Menezes received his $\mathrm{PhD}$ on electrical and computer engineering, with a specialization in informatics, from the University of Coimbra, Portugal, in 2007. Having been an associate researcher at LAAS-CNRS, Toulose, France, he is currently a tenured professor with the University of Coimbra and a senior researcher with the Institute of Systems and Robotics, in Coimbra, Portugal (Email: paulomenezes@ isr.uc.pt).

Article submitted 2021-07-12. Resubmitted 2021-10-12. Final acceptance 2021-10-29. Final version published as submitted by the authors. 